This document is confidential and is proprietary to the American Chemical Society and its authors. Do not copy or disclose without written permission. If you have received this item in error, notify the sender and delete all copies.

\title{
Inherent Driving Force for Charge Separation in Curved Stacks of Oligothiophenes
}

\begin{tabular}{|r|l|}
\hline Journal: & The Journal of Physical Chemistry \\
\hline Manuscript ID: & jp-2014-10866c.R2 \\
\hline Manuscript Type: & Special Issue Article \\
\hline Date Submitted by the Author: & 21 -Jan-2015 \\
\hline Complete List of Authors: & $\begin{array}{l}\text { Wu, Qin; Brookhaven National Laboratory, Center for Functional } \\
\text { Nanomaterials }\end{array}$ \\
\hline
\end{tabular}




\title{
Inherent Driving Force for Charge Separation in Curved Stacks of Oligothiophenes
}

\author{
Qin $W u^{*}$ \\ Center for Functional Nanomaterials, Brookhaven National Laboratory, Upton, NY 11973 \\ qinwu@bnl.gov
}

\begin{abstract}
Coexistence of high local charge mobility and an energy gradient can lead to efficient free charge carrier generation from geminate charge transfer states at the donor-acceptor interface in bulk heterojunction organic photovoltaics. It is, however, not clear what polymer microstructures can support such coexistence. Using recent methods from density functional theory, we propose that a stack of similarly curved oligothiophene chains can deliver the requirements for efficient charge separation. Curved stacks are stable because of the polymer's strong $\pi$-stacking ability and because backbone torsions are flexible in neutral chains. However, energy of a charge in a polymer chain has remarkably stronger dependence on torsions. The trend of increasing planarity in curved stacks effectively creates an energy gradient that drives charge in one direction. The curvature of these partially ordered stacks is found to beneficially interact with fullerenes. The curved stacks, therefore, are identified as possible building blocks for interfacial structures that lead to efficient free carrier generation in high-performing organic photovoltaic systems.
\end{abstract}




\section{Introduction}

One of the least understood processes in organic photovoltaics (OPV) is how free charge carriers are generated from the geminate charge transfer (CT) states at the donor-acceptor interface $^{1}$. Due to its low dielectric constant, electron-hole pairs in organic materials are strongly bound. However, experiments have shown that charge separation can be efficient with little excess energy in the initial CT states $^{2,3}$, i.e., formed by direct excitation instead of exciton dissociation. For efficient electron-hole separation, charge delocalization is shown to play important roles both energetically and dynamically. ${ }^{4-6}$ Long-range exciton dissociation can also reduce the Coulomb barrier. ${ }^{7,8}$ However, a suitable inherent driving force is still needed to complete the charge separation. Electrostatics considerations have offered several possible reasons ${ }^{9}$; though they are not specific for conjugated polymers, the most commonly used donor materials in OPV. Recent coarse-grained simulations ${ }^{10}$ suggest that high local charge mobility and an energy cascade are sufficient for high quantum efficiency in bulk heterojunction (BHJ) OPV; however, detailed atomic-scale structural information is not available from such simulations. In general, the energy cascade is attributed to the fact that charges have lower energies in the crystalline polymer domain than the disordered interface with acceptor materials. ${ }^{7}$ High charge mobility in the mixed phase, on the other hand, is thought to exist within the planar segments of otherwise disordered polymer chains. It is, however, not clear how the energy driving force and high charge mobility can be found simultaneously in the same region, as required for efficient charge separation from the CT states. ${ }^{10}$ Identifying polymer microstructures to support both requirements will supply a missing link at the molecular level to the understanding of charge generation mechanisms and thus provide potential design guidance for new OPV materials. 
Structures and electronic properties of conjugated polymers strongly depend on the collective effects of $\pi-\pi$ stacking and backbone torsion, both of which have low energy barriers to structural changes and, therefore, are responsible for the formation of numerous microstructures in polymer materials. ${ }^{11}$ To improve device performances will certainly require more knowledge of how these microstructures work together at larger length scales ${ }^{12,13}$. On the other hand, most of these microstructures are not yet well characterized experimentally, except for the highly ordered crystalline structure, i.e. when the strong $\pi$-stacking dominates and torsion angles become uniform. However, interesting optoelectronic properties of polymers are often due to non-crystalline structures, ${ }^{14}$ which are difficult to be determined experimentally. Ab initio calculations and molecular mechanics simulations have been used to fill in the unknown structural information. Quantum methods, such as those from density functional theory (DFT), are especially important because they can connect molecular structures with optoelectronic properties. Unfortunately it is still impossible to explore all microstructures in polymeric materials because the polymer conformation space is too large, not to mention the high computational cost of quantum methods. Hence our strategy is to focus on identifying those structures that are mechanistically significant and therefore can be used as building blocks in further understanding or simulation of device performances.

In this work we use the most advanced development from DFT to explore a particular microstructure of conjugated polymers that is able to provide an energy gradient for charge redistribution and support high charge mobility at the same time. Specifically, our focus is the curved stacks. They resemble the ordered $\pi$-stacking structure but their backbones are not planar. However, they are energetically stable, and each polymer chain is curved in a similar pattern so that multiple chains still form $\pi$-stacks. Using oligothiophene as our model, we will show that curved stacks have an inherent bias for charge distribution, and it comes from the nonuniform 
torsions and the strong energy dependence of the charged state on the backbone planarity. The curved stacks also to a large extent maintain local orders and thus can have high local charge mobility. Therefore, they are good candidates for interfacial structures that support efficient charge separation, which is further augmented by the fact that their curvature matches that of a fullerene molecule, the most common acceptor materials in OPVs.

\section{Theoretical basis and computational methods}

All our calculations are performed with the Q-Chem 4.2 package $^{15}$ using the $\omega$ B97X-D functional $^{16}$ in the $6-31 G^{*}$ basis set and SG-1 grid. All charged systems are treated with the unrestricted Kohn-Sham scheme. We choose the long-range corrected $\omega$ B97X-D functional to meet two specific challenges when DFT methods are applied to organic electronic materials, namely charge localization and dispersion interactions. ${ }^{17}$ In a recent work, we systematically investigated the charge self localization behavior as predicted by long-range corrected hybrid functionals ${ }^{18}$. We recommend the range parameter to be $0.2 \mathrm{bohr}^{-1}$ for such functionals for its capability of properly describing charge localization in polymer chains without causing excessive spin contamination. The range parameter in $\omega \mathrm{B} 97 \mathrm{X}-\mathrm{D}$ is $0.2 \mathrm{bohr}^{-1} .{ }^{16}$ This functional further incorporates empirical dispersion corrections, ${ }^{19,20}$ and it is optimized for both covalent and noncovalent interactions. Therefore, $\omega$ B97X-D is particular suited for our studies.

We have chose polythiophene as our system. The torsion angle that is central in our discussion is defined as the inter-ring dihedral angle measured by the atoms S-C-C-S. Except for the 2D torsion potential energy surface scan of the trithiophene molecule $\left(\mathrm{T}_{3}\right)$, we use octathiophene $\left(\mathrm{T}_{8}\right)$ in its all-trans conformation (Figure 2a) as our model of a polythiophene chain. $\mathrm{T}_{8}$ is large enough to fully localize a charge, but still small enough that we can computationally afford to 
study a stack of five $\mathrm{T}_{8}$ chains with our DFT method. In the following results and discussion, we will refer to a stack of $n \mathrm{~T}_{8}$ chains simply as $n$-stack, where $n$ ranges from 2 to 5 .

\section{Results}

\section{Potential Energy Surface Scan of Trithiophene Torsions}

Before we go into results for stacks, we demonstrate in this subsection how the energies of neutral and charged polymers depend differently on the backbone planarity. This difference is the key to understanding later results. Previous work on torsions has mostly focused on the torsion effect on charge transfer integral and the consequent charge localization along a polymer chain, i.e. so called torsional defect. ${ }^{21-23}$ We will carefully avoid these defects by exploring the backbone torsions that only deviate slight from planar. Nonetheless, a small torsion deviation in neutral chains can have significant consequences when charges are introduced.

We use the small molecule trithiophene $\left(T_{3}\right)$ for illustration because it is amenable to a thorough potential energy surface (PES) scan around its two torsion angles. We perform the scan near the trans-planar conformation, in which both torsion angles are $180^{\circ}$. These angles are then changed from $135^{\circ}$ to $225^{\circ}$ at an interval of $5^{\circ}$. That is, the largest deviation from $180^{\circ}$ is $45^{\circ}$. At each point, the geometry of the molecule is fully relaxed except for the torsion angles, and we do geometry optimization only for the non-charged state. With energies of all the optimized geometries we produce a 2D-map that shows the potential landscape of the neutral $\mathrm{T}_{3}$ (Figure 1, top). We then do single-point calculations of the cation at each geometry. All the cation energies are collected to make the same 2D-map (Figure 1, bottom). In both maps, we have adjusted the zero to be their respective lowest energy point, and we use eV as the energy unit throughout this work. 

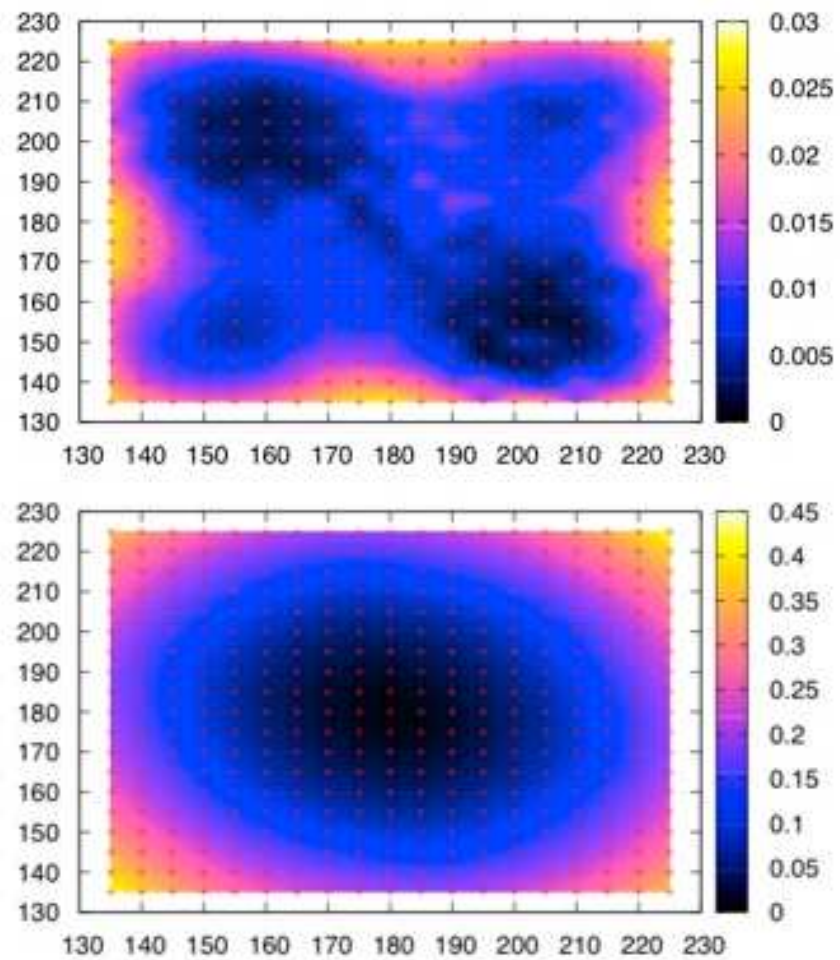

Figure 1. Top: neutral $\mathrm{T}_{3}$ molecule energy (in $\mathrm{eV}$ ) dependence on the backbone torsion angles;

Bottom: $\mathrm{T}_{3}$ cation energy (in $\mathrm{eV}$ ) dependence on the same torsion angles. In both plots, the crosses represent actual calculation results, while interpolated data are used to produce a smooth map. (Plots are made with gnuplot.)

Comparison in Figure 1 reveals important differences between the landscapes of neutral and charged molecules. As the top figure reveals, the neutral molecule actually prefers the torsion angles to deviate slightly from the planar $180^{\circ}$, indicated by the dark color on either the top-left or lower-right part of the neutral map. By having the deviations on neighboring torsion in opposite directions but similar magnitude, the backbone remains linear. A more important feature in the neutral map is that the energy difference is very small, with the highest point being $0.027 \mathrm{eV}$. Therefore all these torsion angles can be explored rather freely in a neutral chain. On the other hand, the cation map has a dark center, meaning the charged molecule undoubtedly prefers a planar backbone with all torsion angles at $180^{\circ}$. More significantly, the energy range in 
the cation map is much wider than that of the neutral map. Its highest energy reaches $0.416 \mathrm{eV}$. This enhanced energy gradient of a cation in a fairly flat PES of the neutral molecule creates a structure-based driving force for charges to move away from less planar polymer segments. While this fact is known, its manifestation in specific polymer microstructures is much less explored and will be the focus of the following subsections.

\section{Formation of a curved stack}

In highly ordered $\pi$-stacks, polymer chains are in a parallel-displaced (PD) configuration, which means instead of being cofacial, atomic positions in neighboring chains have offsets along the long axis. The preference of PD over sandwich (S) configuration in $\pi$ - $\pi$ stacking is already seen in the benzene dimer, and its origin has undergone careful theoretical studies with high-level calculations. $^{24}$ Figure $2 \mathbf{b}$ shows our optimized result of a parallel-displaced $\mathrm{T}_{8}$ 2-stack, confirmed to be the most stable configuration. However, our calculations also find a stable curved configuration of the 2-stack as shown in Figure $2 \mathrm{c}$ that is only $0.065 \mathrm{eV}(1.5 \mathrm{kcal} / \mathrm{mol})$ higher in energy than the PD configuration. The curved stack results from a geometry optimization that uses the planar sandwich configuration as the starting point. Low barrier backbone torsions allow the chains to curve up without much energy penalty. The tendency to remain $\pi$-stacked, on the other hand, forces both chains to curve in a similar pattern so that they remain close to be parallel. For convenience of following discussions, we have defined the back and front of a curve stack as shown in Figure 2c. 


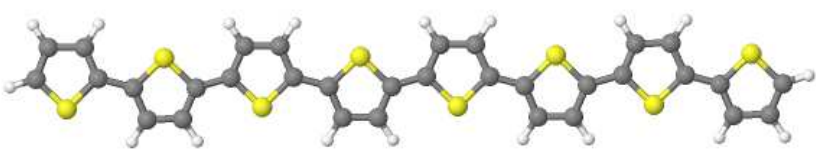

(a)

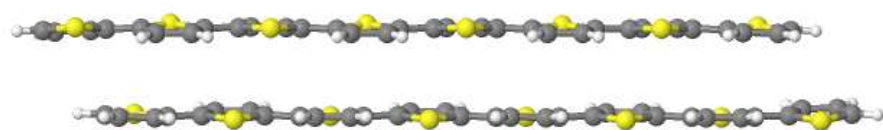

(b)

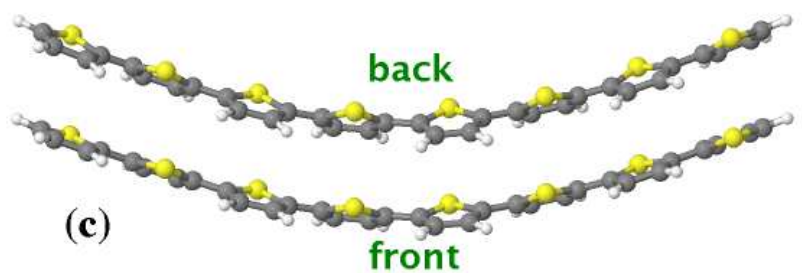

Figure 2. (a) all-trans octathiophene $\left(\mathrm{T}_{8}\right)$; (b) Parallel-displaced configuration of $\mathrm{T}_{8} 2$-stack; (c) curved configuration of $\mathrm{T}_{8} 2$-stack.

Table 1. Comparison of backbone torsion angles in the PD and curved 2-stacks. The angles are listed by their deviation from $180^{\circ}$ (or $-180^{\circ}$ ), in the order from left to right that matches their positions shown in Figure 2.

\begin{tabular}{|l|l|r|r|r|r|r|r|r|}
\hline PD & top & 8.7 & -7.0 & 6.4 & -6.5 & 5.9 & -6.1 & 4.0 \\
\hline & bottom & 4.0 & -6.1 & 5.9 & -6.5 & 6.4 & -7.0 & 8.7 \\
\hline Curved & back & -25.2 & 29.9 & -37.9 & 41.7 & -37.9 & 29.9 & -25.2 \\
\hline & front & -13.3 & 20.0 & -28.2 & 33.4 & -28.2 & 20.0 & -13.3 \\
\hline
\end{tabular}

The bond lengths and bond angles are almost identical between the PD and curved stacks. Their structural difference originates from the backbone torsion. We list all the backbone torsion angles in Table 1. Not surprisingly, the two chains in the PD stack are identical. All their 
torsions deviate from $180^{\circ}$ (or equivalently $-180^{\circ}$ ) only slightly by less than $9^{\circ}$. Deviations in both chains have matching signs and nearly constant magnitude, keeping the chains linear and parallel. Torsions in the curved stack have at least three differences. Firstly, they have larger deviations, though they are still small enough - all less than $45^{\circ}$-- to keep the energy low. Secondly, the deviation magnitude in each chain increases from the ends to the center, effectively creating the curvature. Thirdly, the same torsion always deviates more in the back chain than in the front chain, but the sign remains the same. Therefore, while the two chains manage to be parallel-like, they do not have identical curvatures. These features can also be seen in Figure 3, where the middle three torsions in the curved 2-stack are highlighted.

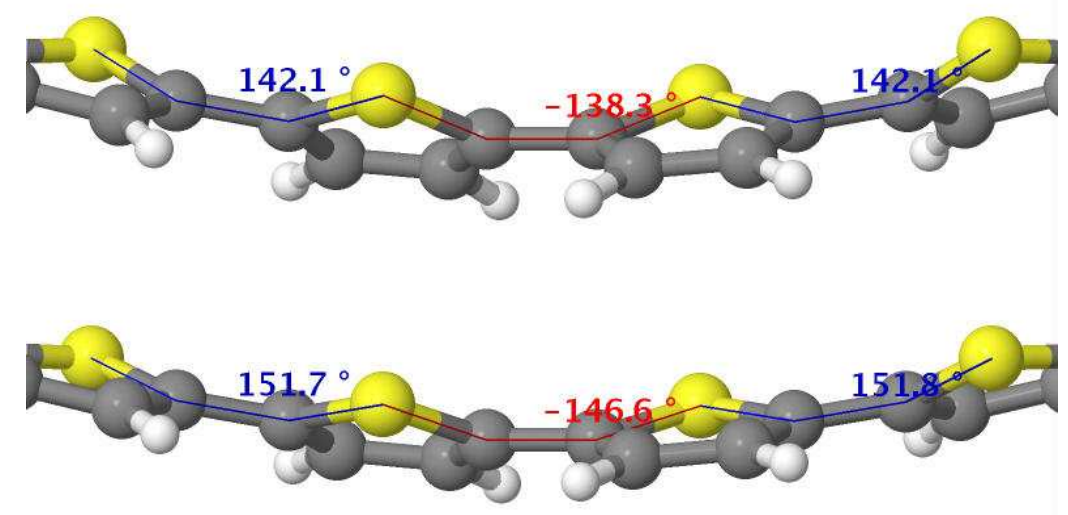

Figure 3. Close examination of the middle three torsions in the curved 2-stack.

To understand how the strong $\pi$-stacking ability might be responsible for the curvature, we compare the highest occupied molecular orbital (HOMO) plots of the PD, curved and sandwich configurations in Figure 4. The geometry of the sandwich configuration is constructed without further optimization by shifting the optimized PD configuration so that conjugated rings become cofacial. The PD preference in $\pi-\pi$ interaction is often analyzed in terms of energies. ${ }^{24}$ But chemical insights and intuitions have also been gained from orbital analysis ${ }^{25}$, i.e. how orbitals from individual chains interact and form delocalized orbitals of the stack. Such interactions 
strongly depend on the lateral displacement and can have strong effects on the electronic coupling parameter in charge transport ${ }^{26}$. Similar to what has been shown before, ${ }^{25}$ the symmetry of individual oligothiophene $\mathrm{HOMO}$ is such that, when two $\mathrm{T}_{8}$ chains are placed cofacially as in the sandwich configuration, phase mismatch causes a nodal plane in the dimer HOMO, which is the antibonding combination of $\mathrm{T}_{8} \mathrm{HOMO}$. Lateral displacement in the PD configuration, however, shifts individual HOMOs from being out of phase to be in phase, thus decreases the antibonding character of the dimer HOMO. Though the corresponding bonding combination is also weakened by the displacement, previous analysis ${ }^{25}$ has shown that the net result is actually an increase of overall bonding, which explains why the PD configuration is more stable than the sandwich configuration. If we turn our attention to the curved configuration, we see a similar phase shifting that removes the nodal plane in the dimer HOMO. That is to say, by using slight backbone torsions that cost little energy, the curved stack creates lateral displacements in opposite directions and becomes stable because of the strong $\pi$-stacking preference. In fact, the curved stack may be effectively viewed as two smaller PD stacks placed against each other at some angle. It is thus understandable why the curved configuration is stable while the sandwich configuration is not.
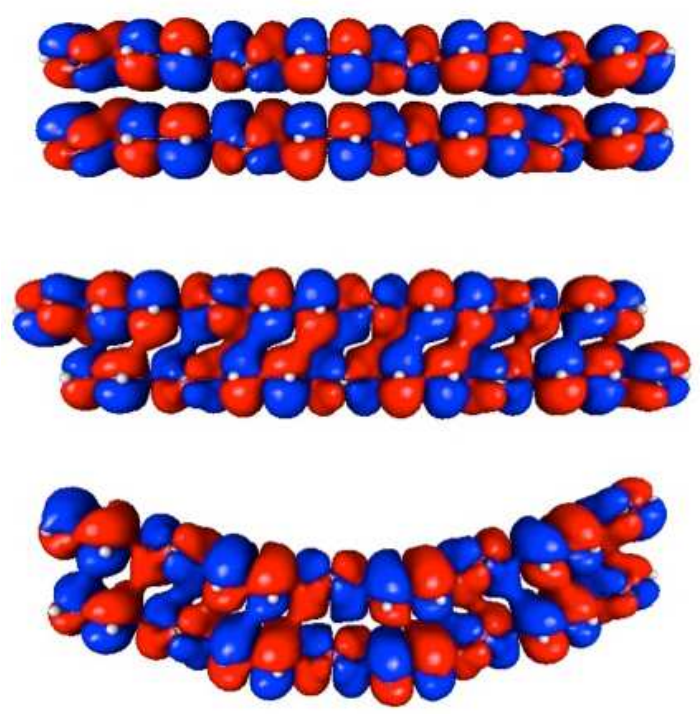
Figure 4. The highest occupied molecular orbital (HOMO) plot for the sandwich (top), paralleldisplaced (center), and curved (bottom) configurations of the 2-stack. The isosurface value is 0.001 (Plots are made with IQmol.)

\section{Curved stacks of larger sizes}

We have extended our calculations to 3,4 and 5-stacks and found stable curved configurations in all of them. In Table 2 we list energies of the curved stacks relative to the optimized PD stack of the same size. While the total energy difference $\left(E_{\text {up }}\right)$ increases with the stack size, the energy difference per pair of neighboring chains -- there are $n$-1 such pairs, -- only varies slightly.

Table 2. Energy differences between optimized PD and curved configurations in $\mathrm{T}_{8}$ stacks. $E_{\text {up }}$ is the relative energy of the curved configuration to the PD configuration of the same size.

\begin{tabular}{|l|l|l|l|l|}
\hline Stack size & 2 & 3 & 4 & 5 \\
\hline$E_{\text {up }}(\mathrm{eV})$ & 0.065 & 0.142 & 0.213 & 0.292 \\
\hline$E_{\text {up }} /(n-1)(\mathrm{eV})$ & 0.065 & 0.071 & 0.071 & 0.073 \\
\hline
\end{tabular}



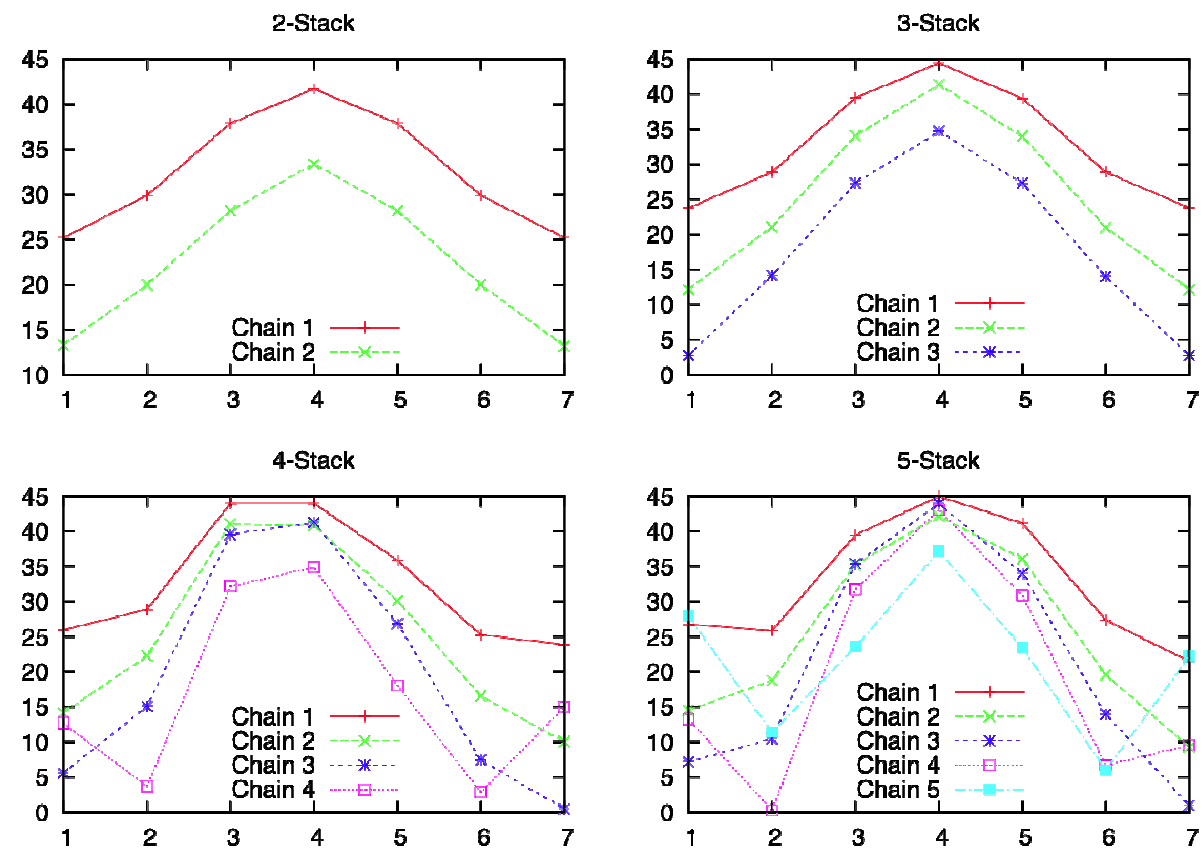

Figure 5. The absolute deviation from $180^{\circ}$ for every backbone torsion angle in the curved 2-, 3, 4- and 5-stacks. Chains are numbered from the back to the front of a stack. Within a chain, torsion angles are numbered from end to end as 1 to 7 , which is used as the x coordinate. (Plots are made with gnuplot.)

We next examine the torsion angles in curved stacks by comparing their absolute deviations from $180^{\circ}$. As shown in Figure 5, there is a general trend of decreasing deviation from the back to the front of the stack. Such a trend is very clear in the 2- and 3-stacks. For 4- and 5-stacks, the increased system size makes geometry optimization more difficult; hence the torsion angle differences have some variations, especially for torsions near chain ends. However, the same trend of increasing planarity for chains on the front is still true.

\section{Charge distribution in cation curved stacks}

Because the cation energy depends strongly on the torsion angles, the fact that chains in a curved stack become increasingly planar from back to front suggests that there is an energy 
gradient for charge distribution when a hole is created in the curved stack, a point that we will probe in this subsection. We performed single point cation calculations of every curved and PD stack. That is to say, a hole is injected into each optimized neutral stack structure without relaxing the geometry. In Table 3 we compare charge distributions in curved and PD stacks using the Mulliken charge population of each chain. For curved stacks, the order is from back to front as defined in Figure 2c. PD stacks are ordered correspondingly. For stacks of more than 2 chains, there is a difference between surface and bulk chains. The former are exposed to vacuum and the latter are sandwiched by other chains. Charges will be more stabilized in bulk due to additional polarization. Such preference is clearly seen in the 3 -stacks, where more than $75 \%$ of the charge is on the bulk chain in both curved and PD stacks. To isolate the effect solely due to molecular structures, we should compare chains positioned similarly in the stack. Therefore, in the 2-stack where both chains are on surface, the front chain in the curved stack takes $66 \%$ of the charge population, as compared to $50 \%$ in a PD configuration. In the 4 - and 5-stacks, where there are 2 and 3 bulk chains, respectively, a clear preference for the charge to be on the front side is also observed in curved stacks. This difference between curved and PD stacks is further illustrated in Figure 6 using the electron density difference between the cation and the ground state of the curved 4-statck.

Table 3. Charge distributions (in percentages) of each chain in different cation stacks. The total charge is +1 in every stack. Numbers from left to right, separated by a dash, are ordered by the chain's position from back to front in the curved stack.

\begin{tabular}{|l|l|l|}
\hline Stack size & Curved & Parallel Displaced \\
\hline 2 & $34-66$ & $50-50$ \\
\hline 3 & $13-76-11$ & $12-77-11$ \\
\hline
\end{tabular}




\begin{tabular}{|l|l|l|}
\hline 4 & $3-9-82-6$ & $5-45-45-5$ \\
\hline 5 & $3-4-30-50-13$ & $4-20-44-26-5$ \\
\hline
\end{tabular}
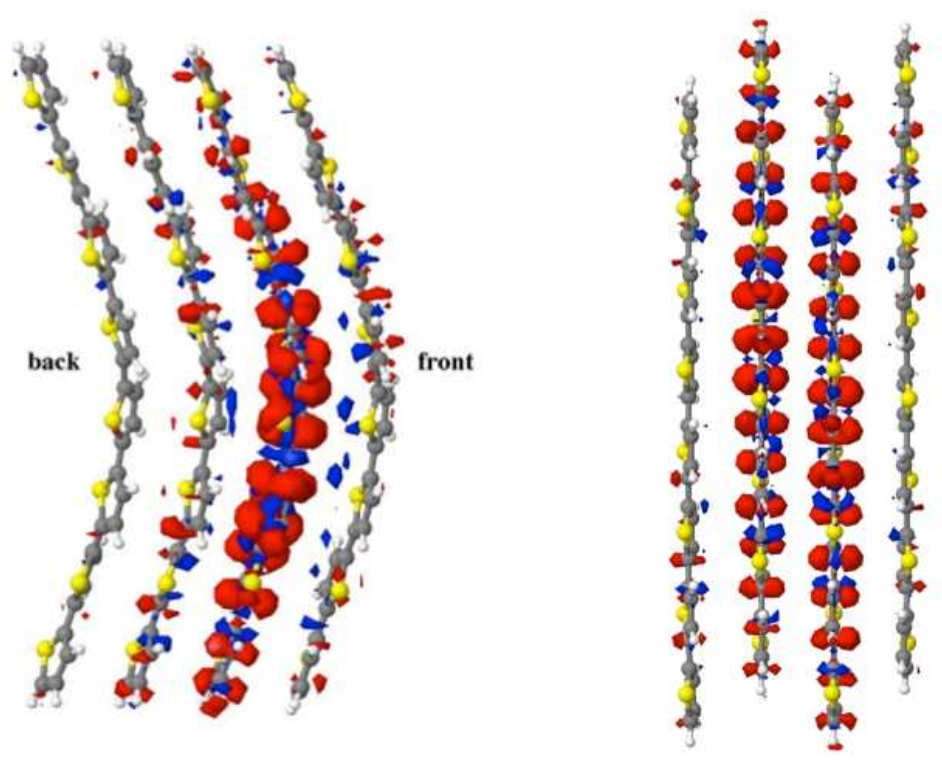

Figure 6. Isosurface plot $\left(0.0005 \mathrm{bohr}^{-3}\right)$ of the density differences between the cation and the ground state of the curved (left) and PD (right) 4-stacks. Red (blue) indicates where the cation has less (more) electron density than the ground state. (Plots are made with Jmol.)

To demonstrate that the biased charge distribution in curved stacks results from the potential landscape created by the increased backbone planarity, we extract the single chain structures from the curved 5-stack and use them to calculate $\mathrm{T}_{8}$ cation energies. We find the cation in the back chain has the highest energy. Cations in the other chains, from back to front in order, are lower in energy by $0.08,0.10,0.13$, and $0.17 \mathrm{eV}$, respectively. We note that this driving force is from pure conformational difference of consecutive chains in a curved stack. While charge 
delocalization and polarization in real materials may alter the energy gradient, they will not change the tilted potential landscape.

\section{Discussion}

\section{On the neglect of polarons}

The biased charge distribution in cation curved stacks is not the same as charge localization. The cations are calculated self-consistently, and therefore include electronic relaxations due to the creation of a hole. How electron relaxes depends on the molecular structure, and the preference of charge distribution is the result of existing torsion angles difference in curved stacks. PD stacks have no such difference and so present no bias for charge distribution. Electronic relaxation is the fast component of polarization. In a slower timescale, charges in conjugated polymers also cause molecular geometry relaxation that become the self-trapping polarons. ${ }^{27}$ Such traps will form regardless of the stacking motif. For instance, when we relax the geometry of the PD cation 4-stack, the charge becomes localized on one of the bulk chains, and bond lengths in this chain become different. Polaron localization by itself does not provide any bias for the charge to move in certain directions. That is, the charge has an equal chance to form a polaron on the other bulk chain in the PD 4-stack. Geometry relaxation in cation curved stacks also leads to charge localization. But the inherent bias in curved stacks makes polarons prefer to be on the front.

Another reason for not considering polaron effects is that charge generation at the donoracceptor interface is an ultrafast ${ }^{28}$ and temperature independent ${ }^{29}$ process, suggesting charges are not localized. Curved stacks have a structure close to that of PD stacks, and therefore can support band-like charge mobility. In fact, HOMOs in neutral curved statcks are completely delocalized. Even the charge distribution in the cation 5-stack also shows significant 
delocalization between the two bulk chains on the same side. The gradual increase of planarity of a curved stack suggests it can smoothly transition into the crystalline domain.

\section{On the effects of side chains}

We have ignored side chains to save computational cost. In real polymer materials, side chains are necessary for solubility and they can have strong effects on polymer conformations. ${ }^{30}$ Including side chains in our calculations, however, is unlikely to qualitatively alter our conclusions. Steric hindrance of side chains makes the parallel displaced stack more stable over the sandwich one, ${ }^{31}$ which is favorable for the curved stacks because they also have the parallel displaced configuration. Moreover, the origin of the inherent driving force comes from the difference in energy dependence on backbone torsions between neutral and charged polymers. Side chains are not expected to affect this difference.

\section{On the significance in bulk heterojunctions}

The curved stacks have a curvature to spatially accommodate a fullerene molecule, which makes the curved stacks favorable at the interface or mixed region with acceptor materials. To demonstrate this point, we performed geometry optimization for the system of $\mathrm{T}_{8} 2$-stack/ $\mathrm{C}_{60}$ and the resulted structure is shown on left side of Figure 7. The oligomer chains are clearly curved in this structure, and the middle panel demonstrates conformation difference between the two chains. We believe that in the presence of $\mathrm{C}_{60}$, the curved stacks will naturally form. In fact, when we put a single $T_{8}$ chain next to $C_{60}$, geometry optimization leads to a curved $T_{8}$ chain, which can serve as the seed for a curved stack. It is then interesting to note that the fullerene sits on the back of the curved stack, while the bias is for the charge to go to the front because the front chain has smaller torsion deviations. Calculations on extracted single chain structures from the 2 -stack $/ \mathrm{C}_{60}$ show the cation energy is $0.11 \mathrm{eV}$ lower in the front chain. This inherent bias in 
curved stacks thus serves as a driving force for charge separation. A system of two chains and one acceptor molecule is of course too small to study charge separation. But one can imagine an interface structure as sketched on the right side of Figure 7, where a stack of polymer chains with decreasing curvature carries the hole away from the interface and smoothly into the crystalline domain. We are doing quantum calculations of larger model systems so as to quantify the energy gradient effects. A model with multiple donors and acceptors will also allow us to find out whether the interface CT states are delocalized and weakly bound, another factor contributing to efficient charge separation. ${ }^{3}$
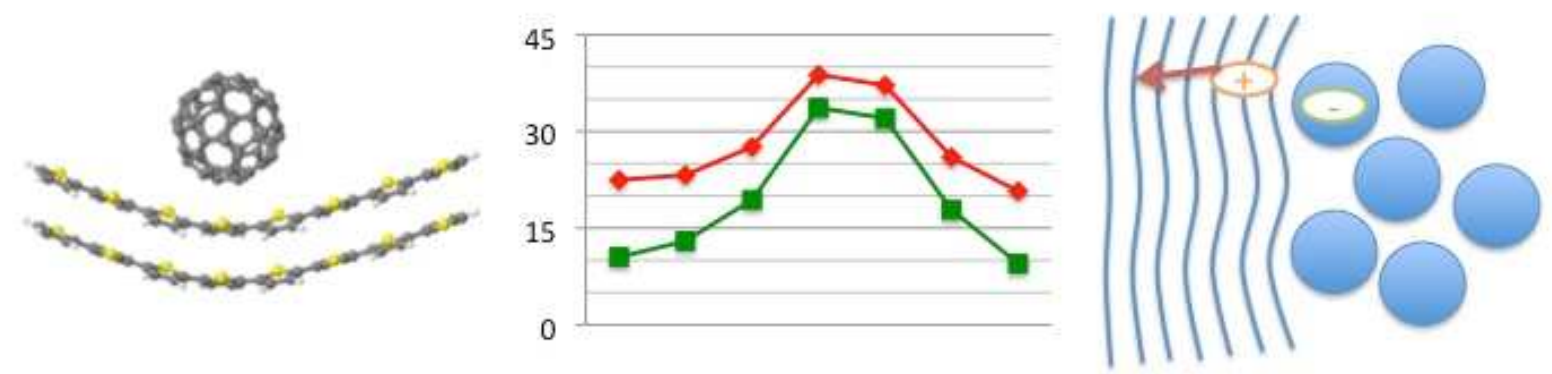

Figure 7. Left: optimized structure of $2-\mathrm{T}_{8} / \mathrm{C}_{60}$. Center: Absolute torsion deviation from $180^{\circ}$ in the chain that is closer to (red diamonds) and away from (green squares) the fullerene. Right: proposed interface structure that supports high efficiency charge separation.

We have focused on one mechanism for efficient charge separation in this work, and that is the coexistence of driving force and high local charge mobility. Specifically, we have provided a molecular basis of such coexistence. Our work does not exclude other mechanisms, including the role of electrostatics ${ }^{9}$ and long-rang exciton dissociation. ${ }^{8}$ All these effects probably have to work together in complex systems like OPV. While our calculations have the apparent limitation of system size, they provide detailed molecular level understanding that cannot be gained in other ways. Therefore our work should be viewed as the part of the smallest scale in a multiscale approach, and our results can be used as building blocks for other simulations. ${ }^{7,10}$ 


\section{Conclusion}

DFT calculations have revealed a stacking motif for polythiophenes different from the most common parallel-displaced $\pi-\pi$ stacking. Low-barrier backbone torsions and polythiophene's strong $\pi$ stacking ability lead to stable curved stacks. Our calculations further demonstrate that curved stacks possess properties that satisfy certain requirements for efficient charge separation in organic photovoltaics. Their structures are sufficiently ordered to support high charge mobility. At the same time, gradual increasing of the backbone planarity in consecutive chains provides an energy gradient for charges to move in a certain direction, one that is further shown to be away from the interface between curved stacks and fullerenes. Curved stacks, therefore, may well be an important polymer microstructure in the donor/acceptor mixed region of highperforming bulk heteorojunction OPV systems, where structural information has been difficult to obtain.

\section{AUTHOR INFORMATION}

\section{Corresponding Author}

*qinwu@bnl.gov

\section{ACKNOWLEDGMENT}

Research carried out at the Center for Functional Nanomaterials, Brookhaven National Laboratory is supported by the U.S. Department of Energy, Office of Basic Energy Sciences, under Contract No. DE-AC02-98CH10886.

\section{REFERENCES}

(1) Brédas, J.-L.; Norton, J. E.; Cornil, J.; Coropceanu, V. Molecular Understanding of Organic Solar Cells: the Challenges. Acc. Chem. Res. 2009, 42, 1691-1699.

(2) Lee, J.; Vandewal, K.; Yost, S. R.; Bahlke, M. E.; Goris, L.; Baldo, M. A.; Manca, J. V.; 
Voorhis, T. V. Charge Transfer State Versus Hot Exciton Dissociation in Polymer-Fullerene Blended Solar Cells. J. Am. Chem. Soc. 2010, 132, 11878-11880. Vandewal, K.; Albrecht, S.; Hoke, E. T.; Graham, K. R.; Widmer, J.; Douglas, J. D.; Schubert, M.; Mateker, W. R.; Bloking, J. T.; Burkhard, G. F.; et al. Efficient Charge Generation by Relaxed Charge-Transfer States at Organic Interfaces. Nat. Mater. 2013, 13, 63-68.

Deibel, C.; Strobel, T.; Dyakonov, V. Origin of the Efficient Polaron-Pair Dissociation in Polymer-Fullerene Blends. Phys. Rev. Lett. 2009, 103, 036402.

Tamura, H.; Burghardt, I. Ultrafast Charge Separation in Organic Photovoltaics Enhanced by Charge Delocalization and Vibronically Hot Exciton Dissociation. J. Am. Chem. Soc. 2013, 135, 16364-16367.

Gelinas, S.; Rao, A.; Kumar, A.; Smith, S. L.; Chin, A. W.; Clark, J.; van der Poll, T. S.; Bazan, G. C.; Friend, R. H. Ultrafast Long-Range Charge Separation in Organic Semiconductor Photovoltaic Diodes. Science 2014, 343, 512-516. McMahon, D. P.; Cheung, D. L.; Troisi, A. Why Holes and Electrons Separate So Well in Polymer/Fullerene Photovoltaic Cells. J. Phys. Chem. Lett. 2011, 2, 2737-2741. Caruso, D.; Troisi, A. Long-Range Exciton Dissociation in Organic Solar Cells. Proc. Natl. Acad. Sci. U.S.A. 2012, 109, 13498-13502.

Yost, S. R.; Van Voorhis, T. Electrostatic Effects at Organic Semiconductor Interfaces: a Mechanism for "Cold" Exciton Breakup. J. Phys. Chem. C 2013, 117, 5617-5625.

Burke, T. M.; McGehee, M. D. How High Local Charge Carrier Mobility and an Energy Cascade in a Three-Phase Bulk Heterojunction Enable $>90 \%$ Quantum Efficiency. Adv. Mater. 2013, 26, 1923-1928.

Brinkmann, M.; Rannou, P. Molecular Weight Dependence of Chain Packing and Semicrystalline Structure in Oriented Films of Regioregular Poly(3-Hexylthiophene) Revealed by High-Resolution Transmission Electron Microscopy. Macromolecules 2009, 42, 1125-1130.

Beaujuge, P. M.; Fréchet, J. M. J. Molecular Design and Ordering Effects in $\pi$ Functional Materials for Transistor and Solar Cell Applications. J. Am. Chem. Soc. 2011, 133, 20009-20029.

Noriega, R.; Rivnay, J.; Vandewal, K.; Koch, F. P. V.; Stingelin, N.; Smith, P.; Toney, M. F.; Salleo, A. A General Relationship Between Disorder, Aggregation and Charge Transport in Conjugated Polymers. Nat. Mater. 2013, 12, 1038-1044.

Reid, O. G.; Pensack, R. D.; Song, Y.; Scholes, G. D.; Rumbles, G. Charge Photogeneration in Neat Conjugated Polymers. Chem. Mater. 2014, 26, 561-575. Shao, Y.; Gan, Z.; Epifanovsky, E.; Gilbert, A. T. B.; Wormit, M.; Kussmann, J.; Lange, A. W.; Behn, A.; Deng, J.; Feng, X.; et al. Advances in Molecular Quantum Chemistry Contained in the Q-Chem 4 Program Package. Mol. Phys. 2014, 1-32.

Chai, J.-D.; Head-Gordon, M. Long-Range Corrected Hybrid Density Functionals with Damped Atom-Atom Dispersion Corrections. Phys. Chem. Chem. Phys. 2008, 10, 6615. Cohen, A. J.; Mori-Sánchez, P.; Yang, W. Challenges for Density Functional Theory. Chem. Rev. 2011.

Shao, N.; Wu, Q. Charge Self-Localization in $\pi$-Conjugated Polymers by Long Range Corrected Hybrid Functionals. Phys. Chem. Chem. Phys. 2014, 16, 6700. Wu, Q.; Yang, W. Empirical Correction to Density Functional Theory for Van Der Waals Interactions. J. Chem. Phys. 2002, 116, 515.

Grimme, S. Semiempirical GGA-Type Density Functional Constructed with a Long- 
Range Dispersion Correction. J. Comput. Chem. 2006, 27, 1787-1799.

(21) Grozema, F. C.; van Duijnen, P. T.; Berlin, Y. A.; Ratner, M. A.; Siebbeles, L. D. A. Intramolecular Charge Transport Along Isolated Chains of Conjugated Polymers: Effect of Torsional Disorder and Polymerization Defects. J. Phys. Chem. B 2002, 106, 77917795.

(22) Darling, S. B. Isolating the Effect of Torsional Defects on Mobility and Band Gap in Conjugated Polymers. J. Phys. Chem. B 2008, 112, 8891-8895.

(23) Cheung, D. L.; McMahon, D. P.; Troisi, A. A Realistic Description of the Charge Carrier Wave Function in Microcrystalline Polymer Semiconductors. J. Am. Chem. Soc. 2009, 131, 11179-11186.

(24) Sherrill, C. D. Energy Component Analysis of $\pi$ Interactions. Acc. Chem. Res. 2013, 46, 1020-1028.

(25) Lutz, P. B.; Bayse, C. A. Orbital-Based Insights Into Parallel-Displaced and Twisted Conformations in П-П Interactions. Phys. Chem. Chem. Phys. 2013, 15, 9397.

(26) Brédas, J.-L.; Beljonne, D.; Coropceanu, V.; Cornil, J. Charge-Transfer and EnergyTransfer Processes in П-Conjugated Oligomers and Polymers: a Molecular Picture. Chem. Rev. 2004, 104, 4971-5004.

(27) Bredas, J. L.; Street, G. B. Polarons, Bipolarons and Solitons in Conducting Polymers. Acc. Chem. Res. 1985, 18, 309-315.

(28) Piris, J.; Dykstra, T. E.; Bakulin, A. A.; Loosdrecht, P. H. M. V.; Knulst, W.; Trinh, M. T.; Schins, J. M.; Siebbeles, L. D. A. Photogeneration and Ultrafast Dynamics of Excitons and Charges in P3HT/PCBM Blends. J. Phys. Chem. C 2009, 113, 1450014506.

(29) Pensack, R. D.; Asbury, J. B. Barrierless Free Carrier Formation in an Organic Photovoltaic Material Measured with Ultrafast Vibrational Spectroscopy. J. Am. Chem. Soc. 2009, 131, 15986-15987.

(30) Darling, S. B.; Sternberg, M. Importance of Side Chains and Backbone Length in Defect Modeling of Poly(3-Alkylthiophenes). J. Phys. Chem. B 2009, 113, 6215-6218.

(31) Dag, S.; Wang, L.-W. Packing Structure of Poly(3-Hexylthiophene) Crystal: Ab Initio and Molecular Dynamics Studies. J. Phys. Chem. B 2010, 114, 5997-6000. 


\section{TOC graphic}
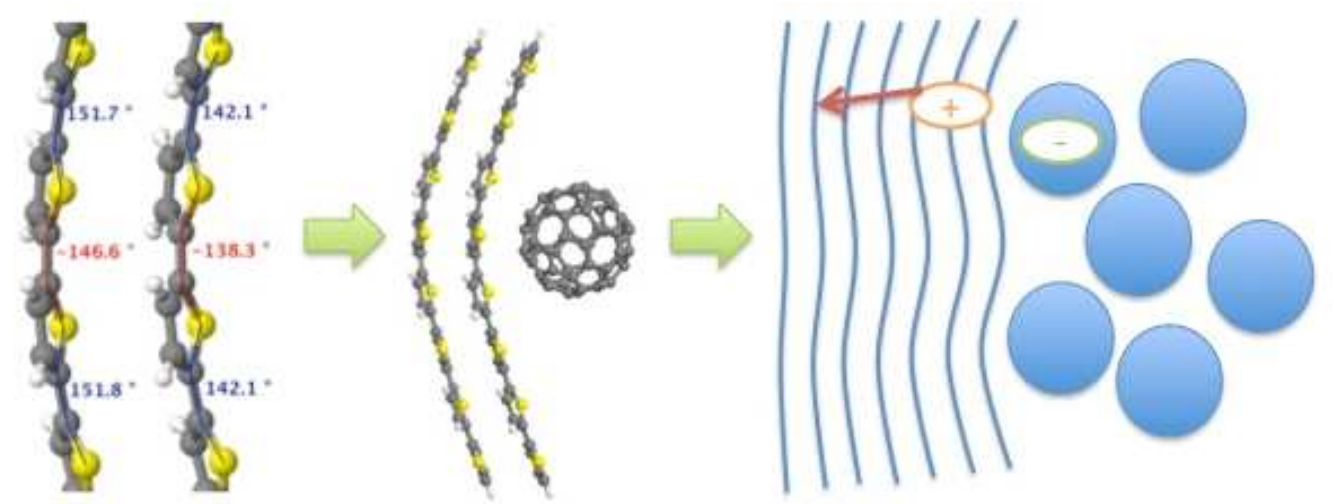

19

20

21

22

23

24

25

26

27

28

29

30

31

32

33

34

35

36

37

38

39

40

41

42

43

44

45

46

47

48

49

50

51

52

53

54

55

56

57

58

59

60 